\title{
Piecewise polynomial phase approximation approach for the analysis of reconstructed interference fields in digital holographic interferometry
}

\author{
Sai Siva Gorthi and Pramod Rastogi \\ Applied Computing and Mechanics Laboratory, Swiss Federale Institute of \\ Technology, 1015 Lausanne, Switzerland. \\ E-mail: pramod.rastogi@epfl.ch
}

\begin{abstract}
This paper proposes a new approach for the analysis of reconstructed interference fields in digital holographic interferometry. In the proposed approach the interference phase to be estimated is conceived as a piecewise polynomial signal; consequently, each segment of the reconstructed interference field is modeled as a polynomial phase signal (PPS) with constant or slowly varying amplitude. Unwrapped phase distribution is then directly computed using the maximum likelihood estimation. Salient features of the proposed approach are: it provides accurate phase estimation from a single record of the interference field; it avoids cumbersome and error prone filtering and 2-D unwrapping procedures; it paves the way to adapt well established PPS analysis tools available in signal processing literature for the phase estimation in holographic interferometry.
\end{abstract}

Keywords: digital holographic interferometry, phase measurement, filtering, parametric estimation, piecewise polynomial approximation, maximum likelihood estimation 


\section{Introduction}

In phase-based techniques in optical metrology, the information on the measurand is encoded in the phase of the recorded interferogram or the fringe pattern. The automated analysis of the fringe pattern to estimate the underlying phase distribution thus plays an important role in many optical measurement techniques. Unlike in classical holographic interferometry, digital holography is known to directly provide the complex amplitude of the reconstructed object wave field. Therefore, usually no separate analysis method is employed for estimating phase in digital holographic interferometry (DHI) [1]. It is simply calculated using the arctan function from the reconstructed interference field. However, phase calculated in this manner is always wrapped and often noisy, making it mandatory to employ filtering and 2-D phase unwrapping procedures $[2,3,4,5,6,7]$. These reasons motivated researchers to develop different strategies or new analysis techniques $[8,9,10,11,12]$.

On the other hand, in many technological applications such as radar, sonar and communications, commonly used signals are often modeled as one-dimensional polynomial phase signals (PPS). In signal processing literature, during the last two decades, many tools have been developed for the analysis of PPS [13]. Unfortunately they are neither directly useful nor have been applied for phase estimation in digital holographic interferometry. With the approach presented in this paper, we intend to open the way for the application of the existing tools in signal processing literature (which were originally developed for the analysis of a particular class of 1-D time signals) to the phase estimation problem in digital holographic interferometry.

This paper introduces a new approach for phase estimation in DHI that directly provides an accurate estimation of the continuous phase distribution from a single record of the interference field. In this approach each row of the reconstructed interference field is divided into a few non-overlapping segments. The data in each segment is modeled as a finite-order polynomial phase signal with constant (or slowly varying) amplitude, embedded in additive complex white Gaussian noise (ACWGN). This approach is accordingly named as piecewise polynomial phase approximation approach (PPPAA/P3A2). The polynomial phase coefficients are computed using the maximum likelihood estimation (MLE) algorithm, reconstruction of which results in the direct estimation of the unwrapped phase distribution. This procedure is repeated for all segments and rows.

\section{Piecewise polynomial phase approximation approach}

Numerical reconstruction of a digital hologram by Fresnel transform results in the direct recovery of the object wave field, which, in general, is represented as:

$$
\Gamma(x, y)=a(x, y) \exp [j \phi(x, y)]
$$

where $a(x, y)$ is the amplitude and $\phi(x, y)$ is the estimated phase of the object wave field. In DHI two digital holograms, one for each state of the object, are recorded (before and 
after deformation). These holograms are reconstructed separately. From the resulting complex amplitudes, $\Gamma_{1}(x, y)$ and $\Gamma_{2}(x, y)$, the interference phase can be calculated by forming the reconstructed interference field as:

$$
\begin{aligned}
A(x, y) & =\Gamma_{2}(x, y) \times \Gamma_{1}^{*}(x, y) \\
& =a_{1}(x, y) a_{2}(x, y) \exp \left\{j\left[\phi_{2}(x, y)-\phi_{1}(x, y)\right]\right\} \\
& =b(x, y) \exp [j \Delta \phi(x, y)]
\end{aligned}
$$

where $\phi_{1}(x, y)$ and $\phi_{2}(x, y)$ are respectively the phases of the object waves before and after deformation. The complex amplitude of the reconstructed interference field in real-world conditions can be expressed as:

$$
A(x, y)=A_{0}(x, y)+\eta(x, y)
$$

where $A_{0}(x, y)$ represents the complex amplitude of the noise-free interference field and $\eta$ represents the noise term. Our aim is to estimate the phase of the actual interference field in equation(3) using P3A2. We propose to divide the reconstructed interference field signal into $N_{w}$ number of non-overlapping segments along each row. Without loss of generality, the problem of interference phase estimation can then be considered as the phase estimation of the following segment of the 1-D signal:

$$
A_{y i}=b_{y i} \exp \left(j \phi_{y i}\right)+\eta_{y i}
$$

where $y, i$ and $\eta$ represent the index of the row, the index of the segment and the ACWGN with zero-mean and variance $\sigma^{2}$, respectively; $y, i$ take values from 1 to $N$ and 1 to $N_{w}$, respectively. For a given row, $A_{i}$ is defined as:

$$
A_{i}=\{A(x)\} \text { for }\left[(i-1) \times N_{s}+1\right] \leq x \leq i \times N_{s}
$$

where $N_{s}=N / N_{w}$. Since the interference phase to be estimated in DHI is, in general, a continuous function of the spatial coordinates, it can be approximated arbitrarily closely, in accordance to Weierstras approximation theorem [14], by a polynomial of a sufficiently high order. Normally, in presence of rapid variations in phase, the order of the polynomial required for accurate representation of $\phi(x)$ will be high. However, by conceiving the phase as a piecewise polynomial signal, modeling the phase locally even by a second-order polynomial can provide quite accurate results. In what follows we represent each segment of the interference field with a second order PPS. Thus the signal to be analyzed can be represented in a simplified form as:

$$
g(x)=b(x) \exp \left[j\left(a_{0}+a_{1} x+a_{2} x^{2}\right)\right]+\eta(x)
$$

The problem of estimating the phase from the noisy observation in equation(3), thus, basically amounts to estimating the parameters like polynomial coefficients $\left\{a_{0}, a_{1}, a_{2}\right\}$ for each segment. We propose to use the maximum likelihood (ML) estimation, an optimal parametric estimation method, for estimating the polynomial coefficients.

The following are the ML estimators [15] for the parameters of the signal in equation(6):

$$
\left(\hat{a}_{1}, \hat{a}_{2}\right)=\arg \max _{a_{1}, a_{2}}\left|\sum_{x=1}^{N_{s}} g(x) \exp \left[-j\left(a_{1} x+a_{2} x^{2}\right)\right]\right|
$$




$$
\begin{aligned}
& \hat{a}_{0}=\text { angle }\left\{\sum_{x=1}^{N_{s}} g(x) \exp \left[-j\left(\hat{a}_{1} x+\hat{a}_{2} x^{2}\right)\right]\right\} \\
& \hat{b}(x)=\operatorname{Re}\left\{g(x) \exp \left[-j\left(\hat{a}_{0}+\hat{a}_{1} x+\hat{a}_{2} x^{2}\right)\right]\right\}
\end{aligned}
$$

where $\hat{a}_{0}, \hat{a}_{1}, \hat{a}_{2}$, and $\hat{b}$ are the ML estimates of $a_{0}, a_{1}, a_{2}$ and $b$ respectively.

Equation(7) indicates that one has to perform a 2-D maximization for implementing the ML algorithm. The estimation is performed in two steps; first, a coarse search, followed by a fine search. Coarse search is done by performing an exhaustive 2-D grid search to find the vicinity of the global maximum. Once this vicinity is found, Nelder-Mead simplex search optimization algorithm [16] is used for refinement. Note that for the 2-D grid search, $a_{1}$ is varied from 0 to $2 \pi$ in steps proportional to $1 / N_{s}$, and $a_{2}$ is varied from 0 to $+2 \pi / N_{s}$ in steps proportional to $1 / N_{s}^{2}$. The fine search algorithm is built using the 'FMINSEARCH' function in the MATLAB, which performs multi-dimensional unconstrained nonlinear minimization by implementing Nelder-Mead simplex search algorithm.

Estimating the coefficient $\hat{a}_{0}$ using equation(8) involves the use of angle function, which will result in wrapping, causing discontinuities at the boundaries of each segment. This can be avoided if $\hat{a}_{0}$ is calculated, for all segments except for the first one, in the following manner:

$$
\hat{a}_{0(i+1)}=\hat{a}_{0 i}+\hat{a}_{1 i} N_{s}+\hat{a}_{2 i} N_{s}^{2}
$$

This modified estimation directly provides the unwrapped phase distribution over the entire row, not just over the individual segments. Figure 1a shows the phase estimated over a segment using the ML estimation procedure; error in estimation is shown in figure $1 \mathrm{~b}$. If $\hat{a}_{0}$ is calculated over all segments using equation(8), the resulting estimated phase of the whole signal is shown in figure 1c. Figure 1d shows the phase of the same signal obtained by calculating $\hat{a}_{0}$ using equation(10). Although the phase estimated along individual rows is unwrapped, to obtain a continuous 2-D phase distribution one additional operation of the type needed to weave all the rows to fall in line one after the other is required. We refer to this operation as phase stitching; this task is as easy as 1-D unwrapping of an ideal wrapped phase map.

\section{Simulation and Experimental Results}

Figure 2a shows the simulated fringe pattern with an $S N R$ of $30 d B$. Phase estimated along the middle row using P3A2 with MLE is shown in figure $2 \mathrm{~b}$. In these simulations the signal is divided into four segments $\left(N_{w}=4\right)$. The estimation procedure is repeated for all rows. Figure 2c shows the 3-D plot of the estimated phase distribution over the entire image.

Regularized phase tracking (RPT) of Servin [10] and windowed Fourier ridge (WFR) of Kemao $[12,11]$ are among the well noted methods in literature which can provide accurate phase estimation from the exponential phase fields. In [17] Kemao et al. 


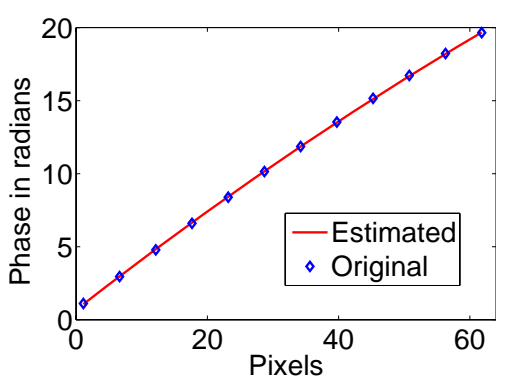

(a)

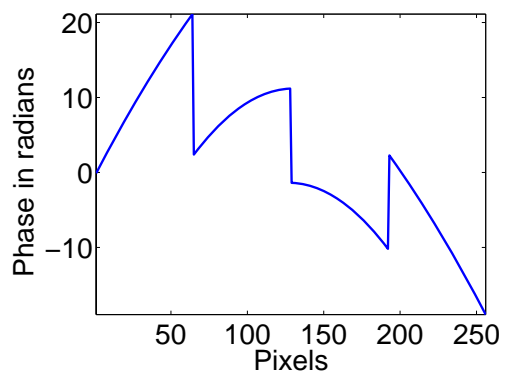

(c)

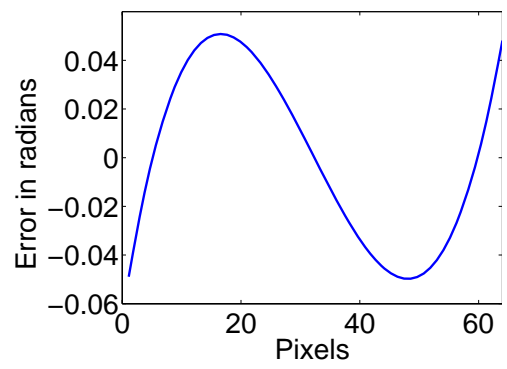

(b)

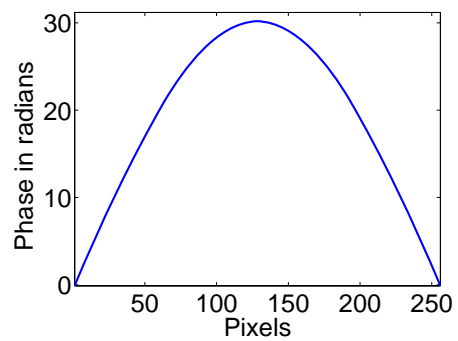

(d)

Figure 1: (a) Estimated phase over a segment using MLE at $S N R$ of $30 d B$ (b) Error in estimation (c) Estimated phase of the whole signal when $\hat{a}_{0}$ is calculated using equation $(8)(N w=4)$, (d) Estimated phase of the same signal when $\hat{a}_{0}$ is calculated using equation $(10)$.

have provided an elegant comparative analysis of five effective wrapped phase filtering techniques which included RPT and WFR. Here we compare the results of the proposed P3A2 with WFR. Figure 3 shows 3-D mesh plots of error distributions when continuous phase distribution corresponding to the pattern shown in figure $2 \mathrm{a}$ is estimated with both the methods. Root mean square errors (RMSE) produced by WFR method (window size of 5 pixels) and P3A2 are given by 0.1325 and 0.0367 , respectively.

The experimental results shown in figure 4 substantiate the effectiveness of the proposed method for the phase estimation in DHI.

On the other hand, the phase map obtained using the arctan function from the reconstructed interference field is shown in figure 5a. For the sake of comparison, the wrapped phase map generated from the continuous phase distribution obtained using the proposed P3A2 method $\left(N_{w}=4\right)$ is shown in figure 5b. A notable improvement in the quality of the phase pattern obtained using the P3A2 method is observed. In order to verify the significance of the piecewise approximation, the wrapped phase map generated from the phase estimated with $N_{w}=1$ (i.e., the entire row is considered as one segment) is shown in figure 5c. It is evident that the estimated phase in this case is having considerable deviation from the actual phase, as the actual phase can be accurately approximated with only 4 th or higher order polynomial. This confirms that the proposed P3A2 method, i.e., with $N_{w}>1$, promises to provide accurate estimation 


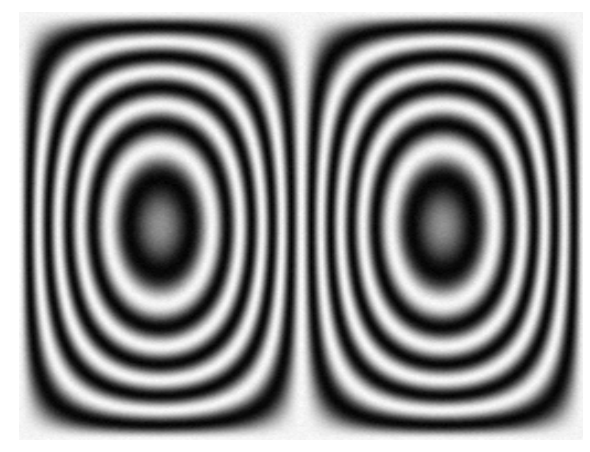

(a)

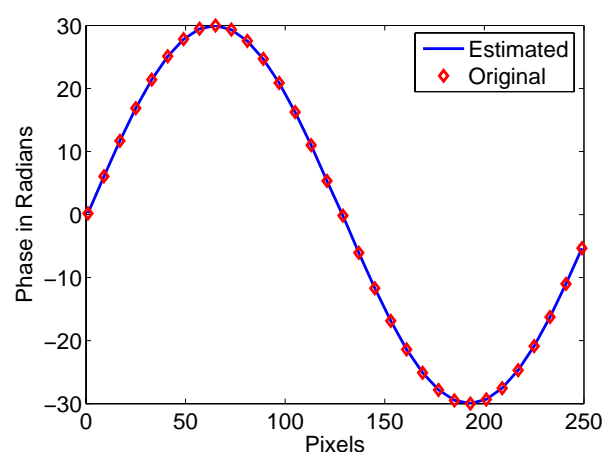

(b)

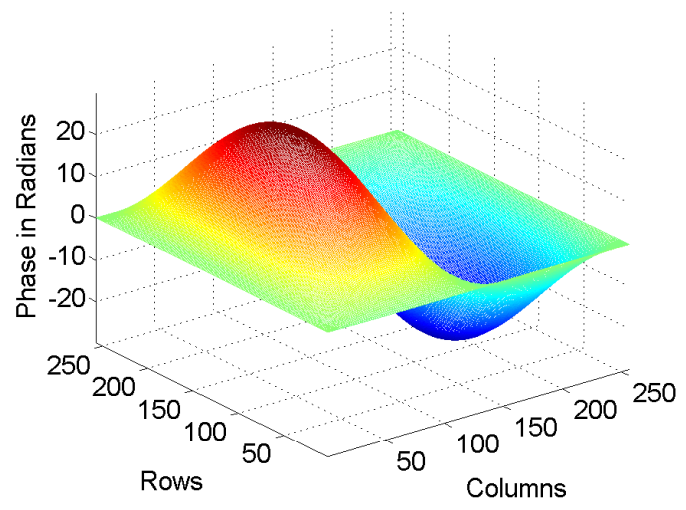

(c)

Figure 2: (a) Simulated fringe pattern at $S N R$ of $30 d B$ (b) Phase estimated along the middle row using the middle row using P3A2 $\left(N_{w}=4\right)$ (c) 3-D plot of the estimated phase over the whole image

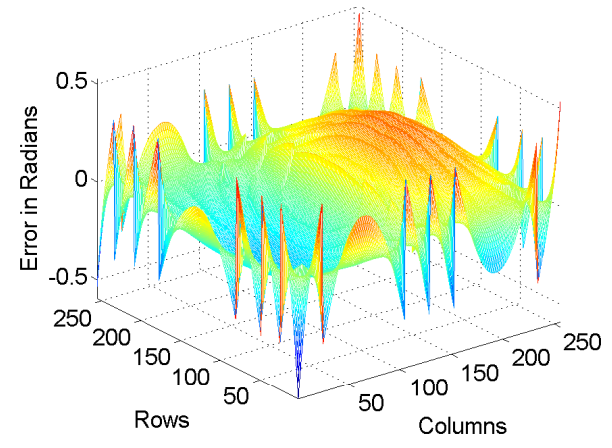

(a)

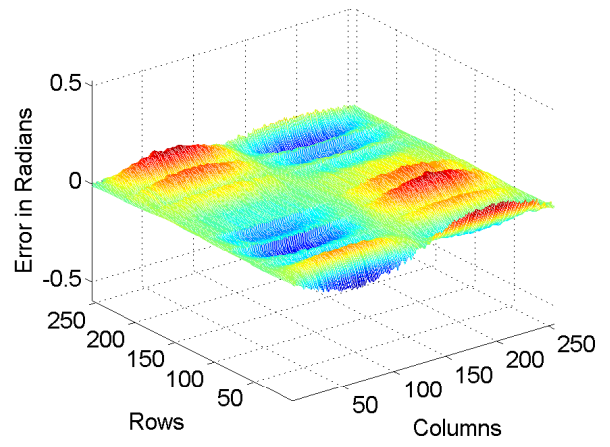

(b)

Figure 3: Error plots when continuous phase distribution of Fig. 2a is estimated with (a) WFR method (b) P3A2 method $\left(N_{w}=4\right)$. 


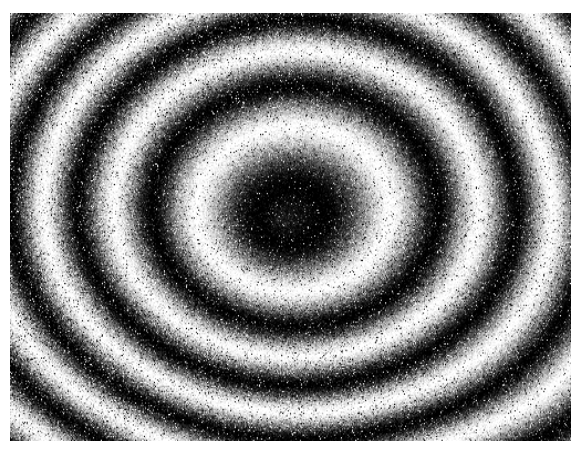

(a)

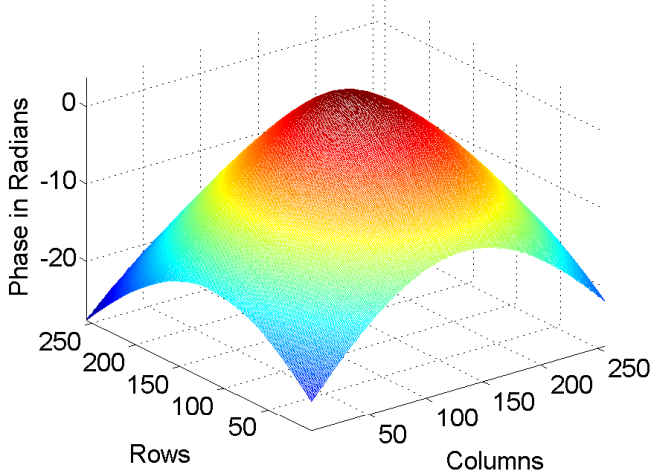

(b)

Figure 4: (a) Experimentally recorded fringe pattern for a circularly clamped object with central loading (b) Phase calculated using the proposed P3A2 method $\left(N_{w}=4\right)$.

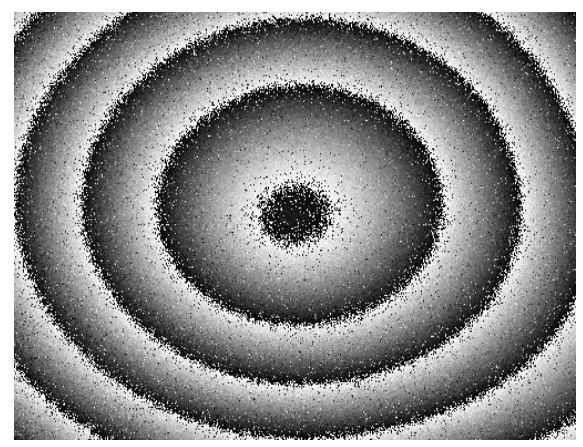

(a)

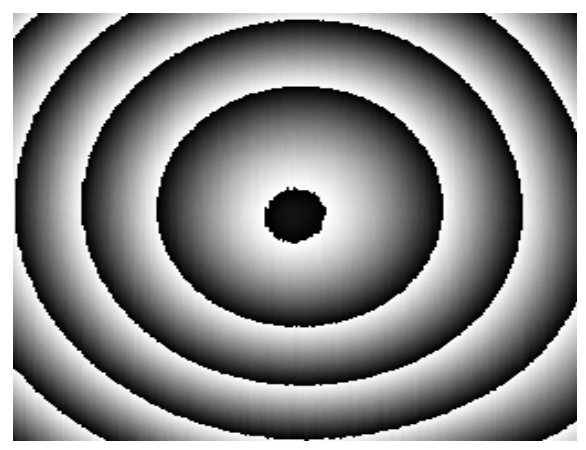

(b)

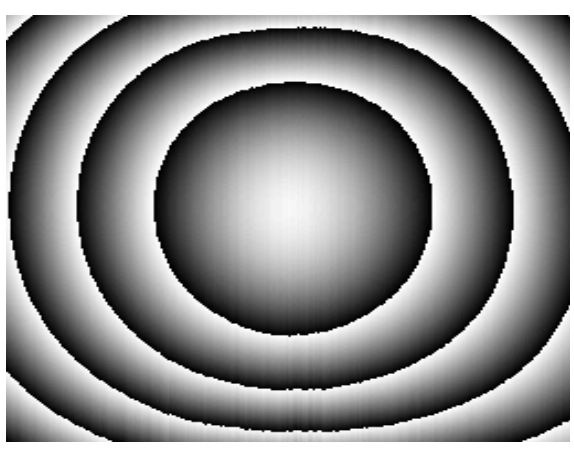

(c)

Figure 5: (a) Phase calculated using the arctan function (b) Wrapped phase generated from Fig. 4b for the sake of comparison (c) Wrapped phase map generated from the estimated phase with $N_{w}=1$. 
even with the lower order polynomial approximation.

An interesting feature of P3A2 is that its performance is unaffected by the absence or the presence of spatial carrier (be it local or global). In applications where the spatial frequency varies strongly within a single image, it only results in the increased value of the coefficient $a_{1}$ (See equation(6)). Therefore, the presented approach can effectively handle the situation without any modifications. But if there are rapid variations in the rate of phase modulation, taking more number of windows (i.e., segmenting the signal into higher number of pieces) will allow P3A2 to accurately approximate, within each segment, the phase of the signal with a polynomial of second-order. The price to be paid for this is additional computational cost. Nevertheless, by developing an adaptive windowing concept that will on-the-fly determine the window length depending on the rate of variations in phase of the data, will allow the proposed approach to make an optimum trade-off between the computational time and the accuracy of estimation in an automated manner.

\section{Conclusions}

To conclude, we have presented a new approach for phase estimation in digital holographic interferometry. Simulation and experimental results have validated the ability of the proposed P3A2 method coupled with the MLE in providing an accurate estimation of the continuous phase from a single record of the reconstructed interference field. Further it does not require 2-D phase unwrapping or any filtering steps. In this paper the implementation of P3A2 is realized with MLE; but in principle any other parametric PPS analysis method can be adapted to expedite the processing. Thus, the proposed approach paves the way to adapt well established PPS analysis tools available in signal processing literature for the phase estimation in digital holographic interferometry.

\section{Acknowledgments}

This work is funded by Swiss National Science Foundation under Grant 200020-113196.

\section{References}

[1] U. Schnars and W. P. O. Juptner. Digital recording and numerical reconstruction of holograms. Meas. Sci. Tech., 13(9):R85-R101, 2002.

[2] F. Palacios, E. Gonc?alves, J. Ricardo, and J. L. Valin. Adaptive filter to improve the performance of phase-unwrapping in digital holography. Opt. Commun., 238(4-6):245-251, 2004.

[3] M. J. Huang and W. Sheu. Histogram-data-orientated filter for inconsistency removal of interferometric phase maps. Opt. Eng., 44(4):1-11, 2005.

[4] Gopalakrishna K. Bhat. Hybrid fringe analysis technique for the elimination of random noise in interferometric wrapped phase maps. Opt. Commun., 111(3-4):214-218, 1994.

[5] Z. Wang, J. Zhang, and Z. Zhao. New methods of the filtering the phase noise in the interferometric sar. In Int. Geoscience and Remote Sensing Symp., volume 4, pages 2622-2625, 2004. 
[6] R. Cusack, J. M. Huntley, and H. T. Goldrein. Improved noise-immune phase-unwrapping algorithm. Appl. Opt., 34(5):781-789, 1995.

[7] K. A. Stetson, J. Wahid, and P. Gauthier. Noise-immune phase unwrapping by use of calculated wrap regions. Appl. Opt., 36(20):4830-4838, 1997.

[8] Y. Morimoto, T. Matui, M. Fujigaki, and N. Kawagishi. Effects of window size on accuracy and spatial resolution in windowed phase-shifting digital holographic interferometry. Strain, 44(5):366-373, 2008.

[9] Y. Morimoto, T. Matui, M. Fujigaki, and N. Kawagishi. Subnanometer displacement measurement by averaging of phase difference in windowed digital holographic interferometry. Opt. Eng., 46(2), 2007.

[10] M. Servin, F. J. Cuevas, D. Malacara, J. L. Marroquin, and R. Rodriguez-Vera. Phase unwrapping through demodulation by use of the regularized phase-tracking technique. Appl. Opt., 38(10):1934-1941, 1999.

[11] K. Qian, H. S. Seah, and A. Asundi. Filtering the complex field in phase shifting interferometry. Opt. Engg., 42(10):2792-2793, 2003.

[12] Q. Kemao. Two-dimensional windowed fourier transform for fringe pattern analysis: Principles, applications and implementations. Opt. Las. Engg., 45(2):304-317, 2007.

[13] B.Porat. Digital processing of random signals. Prentice-Hall, 1994.

[14] Walter Rudin. Principles of mathematical analysis. McGraw-Hill, 1976.

[15] Theagenis J. Abatzoglou. Fast maximum likelihood joint estimation of frequency and frequency rate. IEEE Trans. Aero. and Elect. Sys., AES-22(6):708-715, 1986.

[16] J. C. Lagarias, J. A. Reeds, M. H. Wright, and P. E. Wright. Convergence properties of the nelder-mead simplex method in low dimensions. SIAM Journal on Optimization, 9(1):112-147, 1999.

[17] Q. Kemao, L. T. H. Nam, L. Feng, and S. H. Soon. Comparative analysis on some filters for wrapped phase maps. Appl. Opt., 46(30):7412-7418, 2007. 